\title{
COMPARATIVE STUDY OF THERAPEUTIC EFFECT OF DILTIAZEM, NIFEDIPINE AND FELODIPINE IN PATIENTS WITH RAYNAUD'S PHENOMENON
}

\author{
S. Lambova, L. Sapoundjiev \\ Clinic of Rheumatology, Medical University - Plovdiv \\ Reviewed by: Assoc. prof. Zh. Georgieva, PhD
}

\section{SUMMARY}

Calcium channel blockers have been a treatment of first choice for patients with Raynaud's phenomenon (RP) for many years. The aim of the study: The aim of the study is to compare the therapeutic effect and safety of Diltiazem, Nifedipine and Felodipine in patients with primary and secondary RP and to determine their therapeutic effectiveness in patients with severe RP with digital ulcers. Patients and methods: For a period of six months 86 patients with RP were followed up - 19 with systemic sclerosis and 67 with primary RP. The patients are devided into three groups - treated with Diltiazem, Nifedipine and Felodipine. The first group (I) includes 28 patients $(n=28)$ - 6 with scleroderma ( 3 patients with digital ulcers; $n=3)$ and 22 patients with primary RP. They received Diltiazem at the dose of $60 \mathrm{mg} 3$ times daily. The second group (II) includes 26 patients $(n=26)$ - 6 with scleroderma ( 3 patients with digital ulcers; $n=3$ ) and 21 patients with primary $R P$. They received Nifedipine at the dose of $10 \mathrm{mg} 3$ times daily. The third group (III) includes 32 patients $(n=32)-8$ with scleroderma (4 patients with digital ulcers; $n=4$ ) and 24 patients with primary $R P$. They were treated with Felodipine at the dose of $5 \mathrm{mg} 2$ times daily. For evaluation of the therapeutic efficacy of the medications was used VAS $100 \mathrm{~mm}$ for global assessment of the severity of RP from the patient and the physician before the treatment and at the follow up. It was performed capillaroscopy. There were followed up also the adverse events in the course of treatment. Results: At the end of the second week we found significant decrease of values of VAS for the global assessment of severity of RP by the patients and by the doctor in groups of patients I,II and III. In the three groups of patients the frequency and severity of vasospastic episodes was reduced as well as the degree of pain and disability. Capillaroscopy of nail-fold showed incresed blood flow at the end of the second week compared to baseline in the three groups of patients. Healing of digital ulcers we observed in four patients only in the group treated with Felodipine. These three calcium channel blockers that we used in the present study possess good therapeutic effect in patients with RP, which enhances in the sequence: Diltiazem $<$ Nifedipine $<$ Felodipine. This fact is explained with the increase of the vasoselectivity of the medications in this order. In the same sequence enhances the frequency of their side effects. Conclusion: Diltiazem is appropriate choice in patients with mild forms of primary RP. Felodipine heals digital ulcers in patients with systemic sclerosis and severe secondary RP. The advantages of the medication in comparison with the used in these cases Iloprost are its usage as tablet form and the lower price. Nifedipine takes intermediate place between Diltiazem and Felodipine of its therapeutic effect and safety between Diltiazem and Felodipine. As we consider the knowledge that dyhidropiridines improve endothelial function they become the treatment of choice for long-term therapy in patients with systemic sclerosis and secondary RP among the accessible in Bulgaria medications.

Keywords: Raynaud's phenomenon, Diltiazem, Nifedipine и Felodipine.

\section{INTRODUCTION}

Raynaud's phenomenon (RP) is characterised by episodic vasospasm of small arteries and arterioles of the fingers and the toes provoked by exposure to cold and emotional stress. According to the current Eurapean classification it's defined

Address for correspondence:

S. Lambova, Dept of Pharmacology, Clinic of Rheumatology, Medical University, 15 A, "Vasil Aprilov" Str. 4000, Plovdiv, BULGARIA as secondary RP or syndrome Raynaud (when there is underlying disease) and primary RP or morbus Raynaud (when there isn't underlying cause). In rheumatology the most frequent association is with scleroderma - in $90-95 \%$ of the patients. Digital ulcers are common feature in patients with scleroderma and secondary RP. While patients with primary RP are with benign course of the disease and typically digital gangrene are not observed (3).

Calcium channel blockers have been a treatment of first choice for patients with RP for many years. They are vaso- 
dilators with direct effect on vessel smooth muscle and they inhibit platelet activation. The treatment starts with low dose and titrate upward according to therapeutic effect, the level of blood pressure and the toleration (8). Calcium channel blockers from dihydropiridine and benzothiazepine class are used. Diphenylalkylamine class of calcium-channel blockers don't have therapeutic effect in patients with RP (6).

Benzothiazepine class of calcium-channel blockers.

Diltiazem is used at the dose of $30-120 \mathrm{mg}$ three times daily orally. It has good therapeutic effect in patients with RP which is less than the effect of dihydropiridines (15).

Dihydropiridine class of calcium-channel blockers.

Nifedipine is the best studied drug from this group. The dose is $10 \mathrm{mg} 3$ times daily orally and may be increased to $60 \mathrm{mg}$ daily in refractory cases. Other dihydropiridines are also used in patients with RP - Felodipine, Amlodipine, Nicardipine, Isradipine $(3,8)$.

Felodipine possess high vasoselectivity. Its dose is 2,5-10mg twice daily orally (15).

The selectivity of the medications for blood vessel/heart is 7:1 for Diltiazem, 14:1 for Nifedipine and 100:1 for Felodipine (9).

There are data from the literature that dihydropiridines improve endothelial function. This effect is explained with their antioxidant properties. It's found that they decrease the level of free oxygen radicals and reduce nitric oxide degradation $(2,14)$.

Side effects from calcium channel blockers are common flushing, hypotension, dizziness, headache, tachycardia, ankle oedema, constipation etc. Slow-released tablets of calcium channel blockes are tolerated better $(1,3,5,6,7,13,15)$.

\section{THE AIM OF THE STUDY}

The aim of the study is to compare the therapeutic effect and safety of Diltiazem, Nifedipine and Felodipine in patients with primary and secondary RP and to determine their therapeutic effectiveness in patients with severe RP with digital ulcers.

\section{PATIENTS}

In the study are included 86 patients - 19 patients with systemic sclerosis and secondary RP and 67 with primary RP. They are devided into three groups - treated with Diltiazem, Nifedipine and Felodipine. Criteria for inclusion of a patient in the study and treatment with calcium channel blockers is diagnosis systemic sclerosis (according to the criteria of ACR) and primary RP (12). For the diagnose of primary RP are used criteria of Allen and Brown and exclusion criteria of LeRoy and Medsger (11). The patients are women at the age between 20 and 67 years.

Criteria for exclusion $(1,8)$ :

1. Age $<18$ years.
2. Pregnancy and lactation.

3. Chronic congestive heart failure (NYHA II-IV).

4. Ischemic heart diseases: myocardial infarction or unstable angina (for Nifedipine and Felodipine).

5. Sick sinus syndrome, SA- and AV block (II-III), bradycardia (for Diltiazem).

Patients are treated in university clinic of Rheumatology, Plovdiv for a period of six months.

They were followed up at the end of 2nd, 4th week after the beginning of the treatment with calcium channel blockers and once a month in the next 6 months.

The first group (I) includes 28 patients $(\mathrm{n}=28)$ - 6 with systemic sclerosis ( 3 patients with digital ulcers; $n=3$ ) and 22 patients with primary RP. They received Diltiazem at the dose of 60mg 3 times daily. The second group (II) includes 26 patients $(\mathrm{n}=26)-6$ with systemic sclerosis ( 3 patients with digital ulcers; $n=3$ ) and 21 patients with primary RP. They received Nifedipine at the dose of $10 \mathrm{mg} 3$ times daily Nifedipine. The third group (III) includes 32 patients $(\mathrm{n}=32)-8$ with systemic sclerosis (4 patients with digital ulcers; $n=4$ ) and 24 patients with primary RP. They were treated with Felodipine at the dose of $5 \mathrm{mg} 2$ times daily. The patients were treated with calcium channel blocker as monotherapy without another vasodilator in combination.

\section{METHODS}

For evaluation of the therapeutic efficacy of the medications is used VAS $100 \mathrm{~mm}$ (visual analogue scale) for global assessment of the severity of RP from the patient and the physician before the treatment and at the follow up. It was performed nail-fold capillaroscopy. Global assessment of the severity of RP from the patient includes frequency and the severity of vasospastic episodes, the degree of pain and disability, the presence of digital ulcers. Global assessment of the severity of RP from the physician includes the severity of vasospastic episodes, the degree of disability, the presence of digital ulcers (10).

There were followed up also the adverse events in the coarse of treatment.

\section{RESULTS}

In the first group (I) of patients, treated with Diltiazemwe found significant decrease of values of VAS, that show the global assessment of patients for the severity of RP from $75 \pm 6,36$ to $40,17 \pm 4,19(p<0,05)$. The values of VAS, that show the global assessment of the doctor the severity of RP also decreased significantly from $76,60 \pm 6,53$ to $40 \pm 5,27$ $(p<0,05)$. Side effects that were observed in this group are hypotension $(n=3)$, dizziness $(n=4)$.

In the second group (II) of patients, treated with Nifedipine we found significant decrease of values of VAS, that show the global assessment of patients for the severity of RP from 74,42 $\pm 7,39$ на $35,57 \pm 4,31(\mathrm{p}<0,05)$. The values of VAS, that show the global assessment of the doctor the se- 
verity of RP also decreased significantly from $77,11 \pm 7,89$ на $36,11 \pm 2,13(p<0,05)$. Side effects that were observed in this group are flushing $(\mathrm{n}=4)$, hypotension $(\mathrm{n}=5)$, dizziness $(n=5)$, tachycardia $(n=6)$, ankle oedema $(n=3)$. At the end of the second week there was no tendency for healing of digital ulcers in the patients from group I and II. These patients receive venous infusion of Iloprost, which is the treatment of choice in patients with severe RP with digital ulcers secondary to systemic sclerosis (4).

In the third (III) group of patients, treated with Felodipine we found significant decrease of values of VAS, that show the global assessment of patients for the severity of RP from $74,06 \pm 6,40$ to $28,59 \pm 5,11$ ( $p<0,05)$. The values of VAS, that show the global assessment of the doctor the severity of RP also decreased significantly from $76,71 \pm 6,55$ на 29,53 $\pm 6,76(p<0,05)$. At the end of the second week digital ulcer of the four patients exhibit tendency to heal. And at the end of the second month we observe complete healing in the four patients with scleroderma and severe secondary RP (fig.1; fig. 2). Side effects that were observed in this group are flushing $(n=4)$, hypotension $(n=6)$, dizziness $(\mathrm{n}=7)$, tachycardia $(\mathrm{n}=8)$, ankle oedema $(\mathrm{n}=5)$. Ankle oedema is the side effect that the most often causes stopping the treatment.

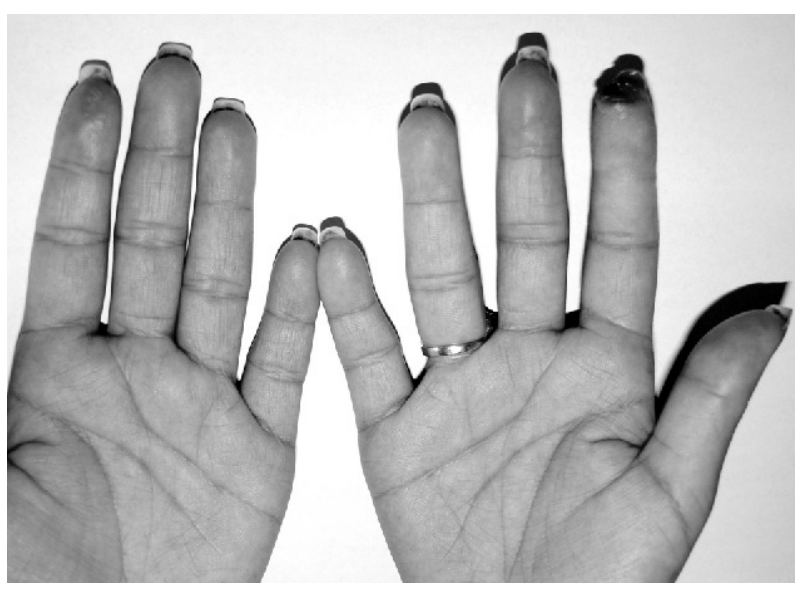

Fig.1. Our patient with systemic sclerosis with digital ulcer-before the treatment with Felodipine.

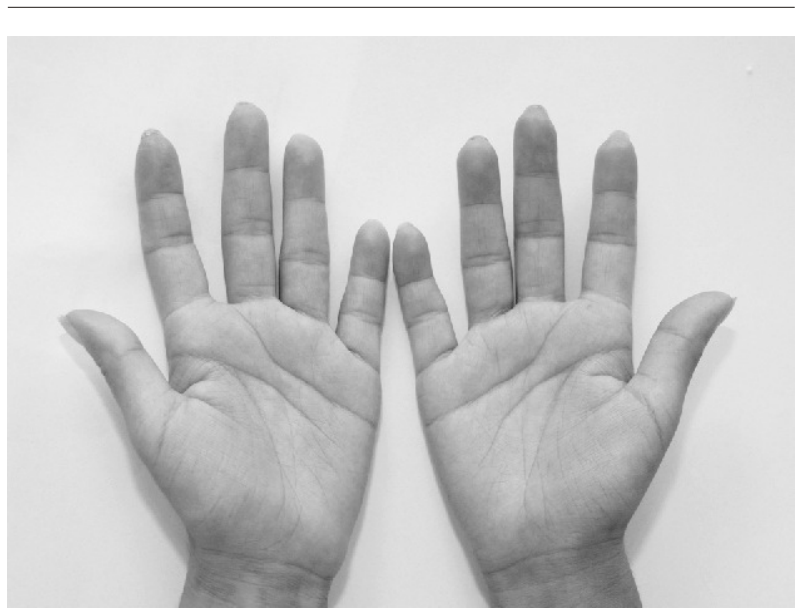

Fig. 2. Two months after the beginning of treatment with Felodipine.
Capillaroscopy of nail-fold showed incresed blood flow at the end of second week compared to baseline in the three groups of patients.

\section{CONCLUSIONS}

1. Diltiazem, Nifedipine and Felodipine decrease significantly the frequency and severity of vasospastic episodes, the degree of pain and disability at the end of the second week after the beginning of the treatment. These medication have good therapeutic effect in patients with RP which enhances in the sequence: Diltiazem $<$ Nifedipine $<$ Felodipine. This fact is explained by the increase of their vasoselectivity in this order.

2. Therapeutic effect of Diltiazem in patients with RP is less than the effect of dihydropiridines - Nifedipine and Felodipine.The frequency of side effects is significantly less in the group of patients treated with Diltiazem.

3. Felodipine healed digital ulcers in patients with systemic sclerosis and severe secondary RP in four observed cases in our study.

4. Nifedipine takes intermediate place of its therapeutic effect between Diltiazem and Felodipine.

5. In the patients from the three groups we observed side effects that are common for calcium channel blockersflushing, headache, dizzines, tachycardia (in groups II and III), ankle oedema. The frequency of side effects increases in the order Diltiazem $<$ Nifedipine $<$ Felodipine.

Diltiazem is appropriate choice in patients with mild forms of primary RP without digital ulcers. Side effects are rare and the medication is with good safety. Felodipine healed digital ulcers in patients with systemic sclerosis and severe secondary RP in four observed cases in our study. The advantages of the medication in comparison with the used in these cases Iloprost are its usage as tablet form and the lower price. Nifedipine takes intermediate place of its therapeutic effect and safety between Diltiazem and Felodipine. As we consider the knowledge that dyhidropiridines improve endothelial function as they increase the level of nitric oxide, they become the treatment of choice for long-term therapy in patients with systemic sclerosis and secondary RP among the accessible in Bulgaria medications. The subject of future studies is comparison of therapeutic effect of Felodipine and Iloprost as well as the combination therapy of these medications in refractory cases.

\section{REFERENCES}

1. Lambev Iv., Uzunov P. References of medicines. Sofia, Medicine and physical culture, 5th ed., 2004:124-127.

2. Berkels R et al. Vascular protective effects of dihydropyridine calcium antagonists. Pharmacol 2003; 69:171-176. 
3. Block JA, Sequeira W. Raynaud's phenomenon. Seminar, Lancet 2001; 357: 2042-2048.

4. Herrick AL, Treatment of Raynaud's phenomenon: new insights and developments. Curr Rheumatol Rep 2003; 5:168-174.

5. Israili $\mathrm{ZH}$. The use of calcium antagonists in the therapy of hypertension in the elderly. Am J Therap 2003; 10:383-395.

6. Leighton C. Drug treatment in scleroderma. Drugs 2001; 61(3):419-427.

7. Ling SM and Wigley FM. Raynaud's phenomenon in older adults Diagnostic considerations and management, Drugs \& Aging, 1999 Sep; 15(3): 183-195.

8. Lisse JR. Raynaud's phenomenon. eMedicine. /www.emedicine.com/med/topic1993.htm - last updated Oct 12, 2004/.

9. Little WC, Cheng CP. Vascular versus myocardial effects of calcium antagonists. Drugs 1994; 47(Suppl 4): 41-5.

10. Merckel, PA. et al. Measuring Disease Activity and functional status in patients with scleroderma and
Raynaud's phenomenon. Arthritis Rheum 2002, 46(9): 2410-2420.

11. Paget SA, Gibofsky A, Beary JF et al. Manual of rheumatology \& outpatient orthopedic disorders: diagnosis and therapy. In: Kirou KA and Crow MK. Raynaud's Phenomenon. 4th edition, Lippincot Williams \& Willkins, 2000: 82-87.

12. Seibold JR., Scleroderma in Kelley's textbook of rheumatology. Philadelphia, ELSEVIER Saunders, 7th edition, 2005:1279-1280.

13. Thompson AE et al, Calcium-Channel blockers for Raynaud's phenomenon in systemic sclerosis. Arthritis Rheum 2001; 44(8):1841-1847.

14. Verhaar MC., Honing MLH, Van Dam Th et al. Nifedipine improves endothelial function in hypercholesterolemia independently of an effect on blood pressure or plasma lipids. Cardiovasc Res 1999; 42: 752-760.

15. Wigley FM. Raynaud's phenomenon. N Engl J Med 2002; 347(13):1001-1007. 